\title{
Space Weather Prediction to enhance the Reliability of the More Electric Aircraft
}

\author{
Jingnan Guo \\ University of Kiel \\ Germany, \\ Email: guo@physik.uni-kiel.de
}

\author{
Giampaolo Buticchi, Chris Gerada \\ University of Nottingham Ningbo China \\ China \\ Email: buticchi@ieee.org \\ chris.gerada@nottingham.edu.cn
}

\begin{abstract}
Power devices manufacturers know that electronic devices are susceptible to radiation events. Galactic Cosmic Rays (GCR) coming from space can interact with the earth atmosphere, generating secondary particles (electrons, neutrons, protons) that can further generate other particles in a cascaded way. When this happen at high altitude, the electronics on-board an aircraft is at risk, because the blocking capability of the semiconductor devices can be momentarily impaired, leading to a single event failure. During solar events The likeliness of such a failure increases and it is related exponentially to the actual voltage across the junction. This paper advocates for a an electrical power distribution system that can adapt its voltage depending on the space weather forecast.
\end{abstract}

\section{INTRODUCTION}

Highly-energetic cosmic charged particles reaching the space environment and avionics near Earth may have radiation effects which can be mission limiting causing a prompt or accumulated degradation of subsystems which ultimately may result in subsystem or system failures, or even catastrophic system anomalies of satellite in operation. Such particles may also propagate further down into Earths magnetosphere and atmosphere and generate secondary particles via atomic and nuclear interactions with Earth's atmosphere as shown in Figure 1. These particles including protons, muons, electrons and most dangerously neutrons, which interact with matter in a a different manner, would cause similar effects on high-altitude electronics and payloads on aircraft whose operational safety is extremely important. This is even more relevant because in the future more electric aircrafts will feature an increased amount of electrical subsystems and power electronics converters. Radiation effects on satellites, spacecraft and payloads include:

- Damage to electronic components due to total ionizing dose (TID), normally induced by electrons and protons via bremsstrahlung. Examples include threshold voltage shifts and leakage currents in complementary metal oxide semiconductors (CMOS).

- Displacement damage for photonics e.g. charged coupled devices (CCD) transfer deficiency, reduction in solar cell efficiency which results in a degradation of the mission efficiency. This is normally caused by non-ionizing energy loss (NIEL) of protons, electrons, neutrons and ions.

- Single event effects (SEE) which cause failures in the memories, microprocessors, latch-up, burnout, gate rupture, transients in op-amps, and comparators. This can be caused by direct ionization by a single heavy ion or nuclear interactions by high-energetic protons and neutrons. SEE in space missions is often mitigated via utilizing redundant components and error detection and correction (EDAC) protected memory.

- Charging due to electrons which can cause phantom commands from electrostatic discharge.

Failures of the above electronic systems caused by space hazards could lead to tremendous economical cost. Governments currently spend over 65 billion Euro a year on space programmes (including space research) leading among other things to the development of modern technologies such as satellite navigation, satellite television, weather forecast, automated piloting, etc. which have become indispensable tools in our everyday life. Therefore, increased scientific knowledge and, based on that, advanced mitigation engineering investigations, are of utmost importance. However, reliable mitigation strategies of such system failures have not yet been fully developed, largely due to the highly-interdisciplinary nature of the problem. This study hints at how a simple integration between these different disciplines could bring benefits in terms of resilience to solar events for the aircraft electrical systems.

\section{Space Weather Modeling}

Radiation damage induced by charged particles from space comes mainly form two sources, Galactic Cosmic Rays (GCRs) and Solar Energetic Particles (SEPs), both composed of mainly protons. The GCR is omnipresent, ubiquitous, and is modulated by the heliospheric activities which generally follow the 11-year solar cycle due to the evolution of the convective magnetic dynamo of the Sun. Broadly speaking, the stronger the solar activity the higher would be the deflection of interplanetary GCRs (generated by e.g., supernova explosions) reaching the solar system, resulting in a reduction of the GCR flux. The temporal evolution of GCR fluxes are relatively regular and have been well studied and understood and we refer the readers to e.g., [1]. The radiation damage induced by GCRs is mainly a long-term accumulative effect (i.e., TID).

On the other hand, solar eruptive phenomena are highly nonlinear, dynamic, imprudent, sporadic, and involve enormous differences of system scales and physics mechanisms and thus are extremely hard to predict, study and evaluate. 


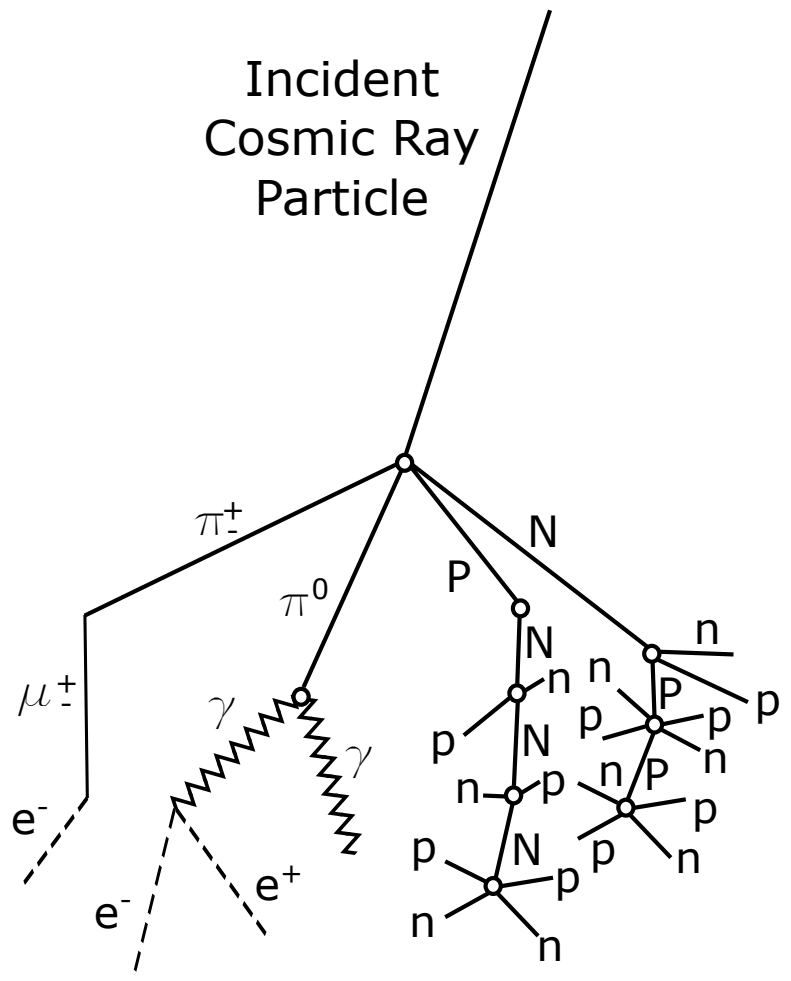

Fig. 1: Atomic and nuclear interactions of cosmic rays with the atomsphere of Earth which creates various secondary particles such as protons, neutrons, muons and electrons.

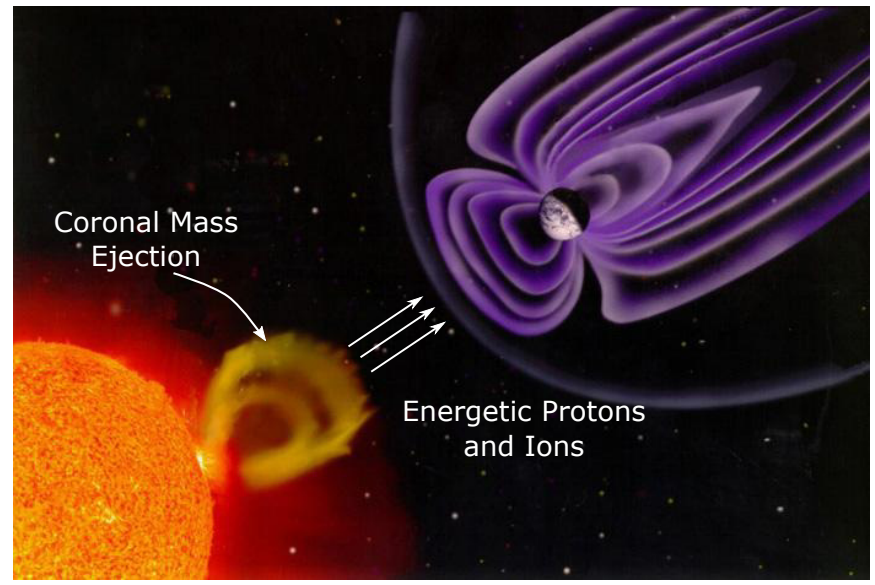

Fig. 2: Acceleration, release and transportation of solar energetic particles. Modified from NASA image.

Intense SEP events can result in very high fluxes and dose rates and despite of being rather infrequent, are sporadic and very unpredictable and could result in severe radiation damages as described in Section I. The resulting radiation effect from a Solar Particle Event (SPE, depicted in 2) is the result of four major procedures:

- (i) the acceleration processes at the Sun which are often related to the flare eruptions such as coronal mass ejections and associated shocks [2],
- (ii) the fluxes and energies of the accelerated particles injected into the open magnetic fields and their interplanetary transportation along as well as across the magnetic fields which are connected to Earth [3], [4],

- (iii) the shielding of the Earth's magnetosphere against such energetic charged particles (which is also the case for GCRs),

- (iv) and finally the atomic and nuclear interactions of particles that have penetrated through the magnetosphere with the atmosphere of Earth and the generation of secondary particles as described in Section I.

The occurrence of a solar flare accompanies a coronal mass ejection (CME) extending outward from the Sun. Due to the extremely fast speed of some CMEs shown as an entrained flux rope compared to the ambient solar wind, a leading shock can often be formed at the front edge of the CME and the compressed sheath plasma behind the shock. Both flares and CME shocks can accelerate charged particles to relativistic energies through different mechanisms such as stochastic mechanism, magnetic reconnection [2] and continuous acceleration at the interplanetary shock front [3]. The accelerated particles are often, but not necessarily, bounded to the magnetic field direction and spiral away from the Sun along these fields. The reason particles can diffuse across (perpendicular to) the magnetic field lines is often related to the turbulent nature of interplanetary magnetic fields and pitch angle scattering of energetic particles [4]. These shock-accelerated ions streaming away from the shock can generate waves that can often be observed prior to the arrival of particles used for forecasting the in-situ onset of potentially hazardous SPEs. For relativistic particles which can travel through 1 astronomical unit within 1020 minutes, this forecast however is not sufficiently early. Therefore observation-based (remote sensing observation of the solar photosphere evolution) modeling tracking the evolution of the solar eruption from its initial trigger to the energy release and particle kinematics, e.g., combining largescale magnetic-hydrodynamic simulations of the solar surface plasma with small-scale particle acceleration simulations, is the essential key to forecast such hazards.

When SEPs reach Earth, they still need to penetrate though Earth's magnetosphere (procedure iii as mentioned above) which has an energy-dependent shielding effect against charged particles (higher energetic particles can penetrate more easily). Also due to the configuration of the magnetosphere, the shielding is more efficient near the equator but rather inefficient near the polar regions where less energetic particles can also stream along the polar fields and enter Earth's atmosphere. Thus radiation hazard for aircraft at high altitudes and high latitudes is a bigger concern than aircraft at other locations. Combining the penetration of particles through Earth's magnetosphere and the interaction of remaining particles through the atmosphere, Monte Carlo codes such as PLANETOCOSMICS [5] can simulate this process in order to predict energetic particle fluxes including the generated secondaries and radiation dose rates at various latitudes and 
different altitudes of the atmosphere.

The above model combined with the input knowledge of particles arriving Earth during eruptive solar events based on both particle measurements on spacecraft near Earth and modeling of particles transported here from the acceleration site at/near the Sun can give important information on potential radiation damage on avionics at these locations.

\section{EFFECTS ON COSMIC RAYS ON THE POWER ELECTRONICS}

The awareness that cosmic rays affect the failure of the silicon power devices is known by academic and industrial researchers. Some manufacturers, like Semikron [6], have included radiation-induce failure rates depending on the blocking voltage in their data-sheets. In-field testing at high altitude or accelerated testing with neutron beams can be adopted to perform accelerated lifetime tests at different voltage beams. Although the high altitude tests give a realistic mix of high energetic particles, the acceleration factor is low, that is why accelerated testing with neutron beams constitute the industry standard. Formulas to interpolate the failure rate at different voltages normally imply the use of an exponential model [6]. Correction factors depending on the temperature [7] and on the altitude are normally provided in the data-sheet. Altitude dependency is particularly relevant for airborne application, as it was highlighted that the radiation intensity increases by a factor 2 every $1000 \mathrm{~m}$ of altitude [8]. During a solar event, the reduced shielding by the atmosphere makes the semiconductors even more susceptible to this problem.

A typical lifetime formula from the data-sheet is reported in equation (1) [7], and three components can be highlighted:

- A bias voltage $V_{D C}$-dependent component, from an inception voltage $C_{1}$ and under the assumption $V_{D C}>C_{1}$.

- A temperature-dependent component.

- An altitude-dependent $h$ component.

$$
\begin{gathered}
\lambda\left(V_{D C}, T_{v j}, h\right)=C_{3} \exp \left(\frac{C_{2}}{C_{1}-V_{D C}}\right) \exp \left(\frac{25-T_{v j}}{47.6}\right) \\
\exp \left(\frac{1-\left(1-\frac{h}{44300}\right)}{0.143}\right)
\end{gathered}
$$

As it is, this model is mainly obtained through the fitting of experimental data, especially for the voltage-dependent part. The altitude-dependent part normally does not consider altitudes above $6000 \mathrm{~m}$ and does not take into account the change in dose rate during solar events.

Several researchers [9], [10] have been investigating the reliability of power converters due to radiation failure. A high energetic particle coming from the atmosphere can hit the junction of a semiconductor power devices, depositing a certain amount of energy. In a device, which at the moment of the event is in blocking state, the electric field distribution is modified, leading to higher values that can trigger the impact ionization. As highlighted in [6] and described [11], neutrons are the most dangerous secondary particles, because they can deposit their energy in a single spot. If the device does not revert to its blocking state after the event, a short-circuit failure that leads to the destruction process normally called singleevent burnout [12].

\section{Active Control of the Power Electronics}

\section{A. Common practices for protect the electronics}

Several approaches are possible regarding the protection of electronic circuits to cosmic rays. For integrated circuits the dangerous condition is represented by neutrons and alpha particles that can change the data of a SRAM register, leading to unpredictable results. One of the adopted ways to reduce this risk is to use mathematical methods, like the errorcorrection-code (ECC), that increases the resiliency to a bit change. Depending on the direction of the particle, one or more SRAM cells can be affected and the degree of the ECC redundancy sets the maximum number of cells that can change simultaneously and still guarantee safe operations. If this limit is exceeded, the error can still be detected to some extent, and the unit can be safely disabled.

For power electronics converters, the usual way to safeguard the operation is to set the semiconductor devices operating point around $65 \%$ of their breakdown voltage. This is generally valid for $\mathrm{Si}$ devices at ground level and leads to an under-utilization of the full voltage capability of the converter. Some manufacturers, like International Rectifier, also offer a portfolio of Rad-Hard MOSFETs in their HiRel series. Because these are very special products, it might still be of interest to use standard devices for airborne application.

Some researchers have been studying that the use of other semiconductor types that can operate closer to their breakdown limits, like $\mathrm{SiC}$, can lead to benefits for high voltage photovoltaic converters [13]. The smaller chip area of the SiC transistors for the same current capability further reduces the probability to be hit by a high energetic particle [13].

For power electronics, the approach of adding some degrees of redundancy can be pursued, to make the overall system more resilient to short-circuit faults. A possibility is to use fault-tolerant power converters, as in Fig. 3 [14], where a series-resonant converter is modified by adding an additional switch in the rectifier stage. In the case of a short-circuit fault, the shorted device is used to carry the current and the rectifier is reconfigured as a voltage doubler, allowing for an unchanged output voltage. The requirements are an increased semiconductors area (in post-fault mode, the current stress is doubled) and an additional device to perform the reconfiguration.

Another possibility is to introduce redundancy at system level, by making the whole Electrical Power Distribution System (EPDS) resilient to faults that impair the converter. For DC distribution systems, a possibility is to use multi-port power converters [15]-[17], that can still operate even if a port is damaged. The EPDS normally has more than one bus, in order to prevent catastrophic failure. The use of multi-port converter would allow having the same functionality allowing 


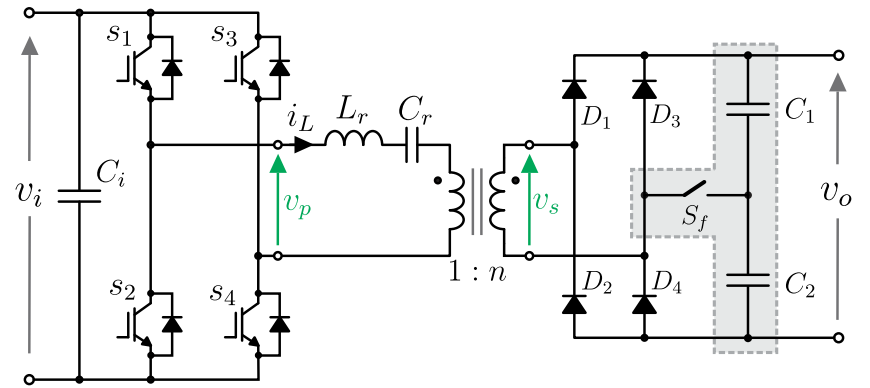

Fig. 3: Fault-tolerant Series Resonant Converter [14].

for the power exchange between the different lines, effectively improving the functionality.

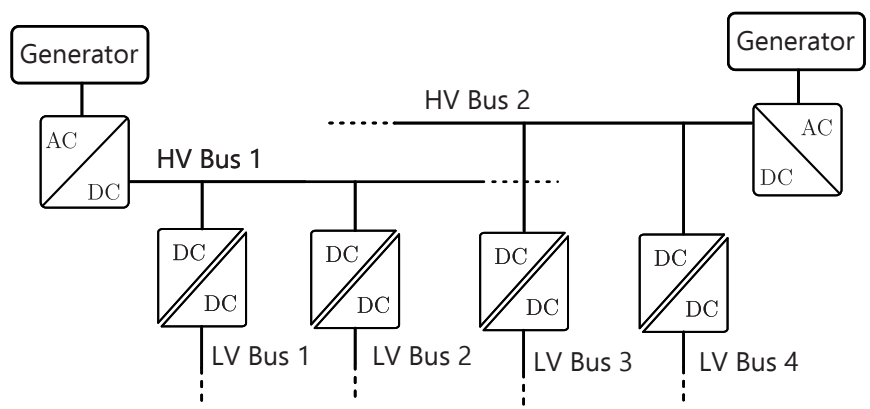

(a)

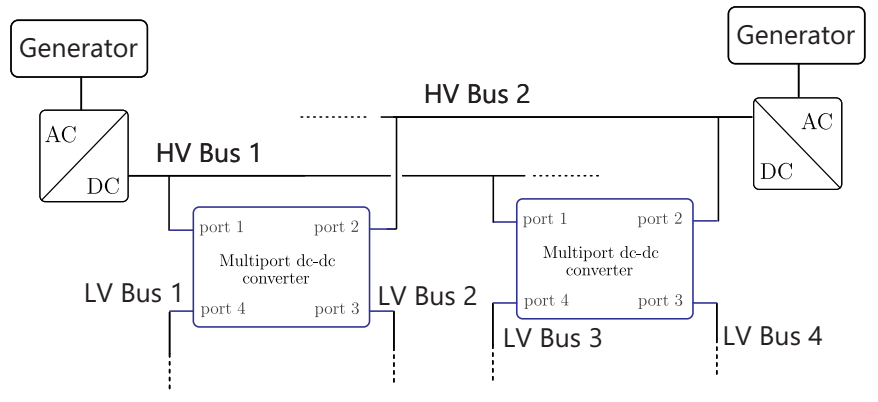

(b)

Fig. 4: Architectures of the Electrical Power Distribution System with single-input single-output DC/DC converter (a) and with multi-port power converters (b) [17].

\section{B. Voltage Control of the EPDS}

Although fault-tolerant topologies allows for resilient operation, it is opinion of the authors that the fault occurrence should be avoided at all costs, since it generates unpredictable transients, possible deterioration of the service and forces the owner of the aircraft to perform the maintenance.

At the moment, because of the small penetration of power electronics in the aircrafts, there are no statistical data of failures happening due to solar events induced radiation. Nevertheless, the interest of the funding agencies on this topic is high, as documented by the numerous call for proposals by the funding agencies. This problem is probably going to become more relevant in the future due to the increase aircraft electrification.

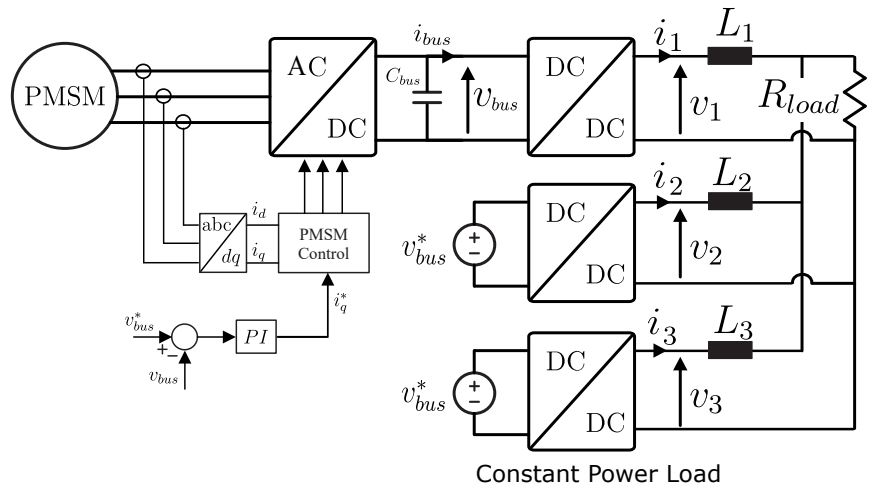

Fig. 5: Example of the EPDS used for the simulations.

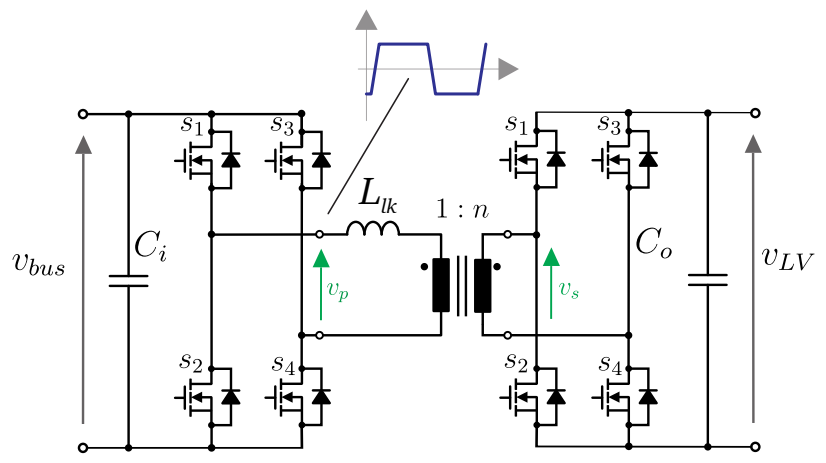

a)

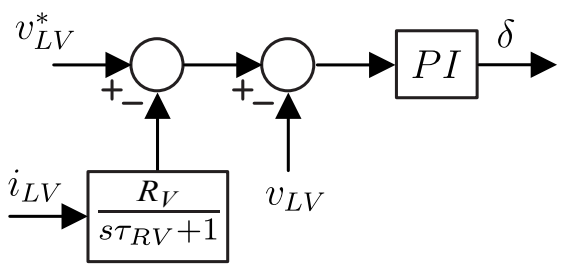

b)

Fig. 6: Schematic of the Dual Active Bridge (DAB) (a) with its voltage control (b).

Since there is an exponential relationship [6] between the blocking voltage and the occurrence of a single-event burnout, with the knowledge of the space weather, the voltage of the whole EPDS can be adapted depending on the radiation risk.

Fig. 5 shows an example of an EPDS where a three-phase inverter is connected to a Permanent Magnet Synchronous Machine (PMSM) that acts as a generator. A Field-Oriented Control (FOC) is adopted to control the bus voltage $v_{\text {bus }}$.

Three DC/DC converters are connected to a LV bus. This kind of grid is used by several researchers to analyze the stability issues of the EPDS [18], [19]. Converters 1 and 2 feed the bus. The choice of the DC/DC converter is the Dual Active Bridge (DAB, see Fig. 6), that has attracted the interest of the industry due to its good performance in terms of softswitching and power control. Fig. 6 shows the topology of the converter and its voltage control. The controlled variable 

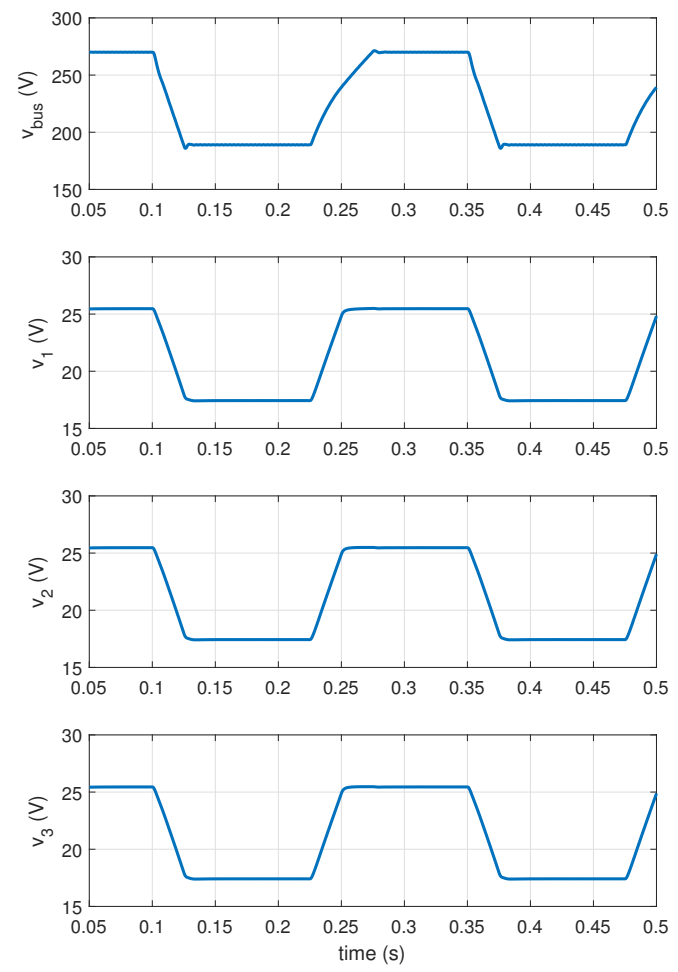
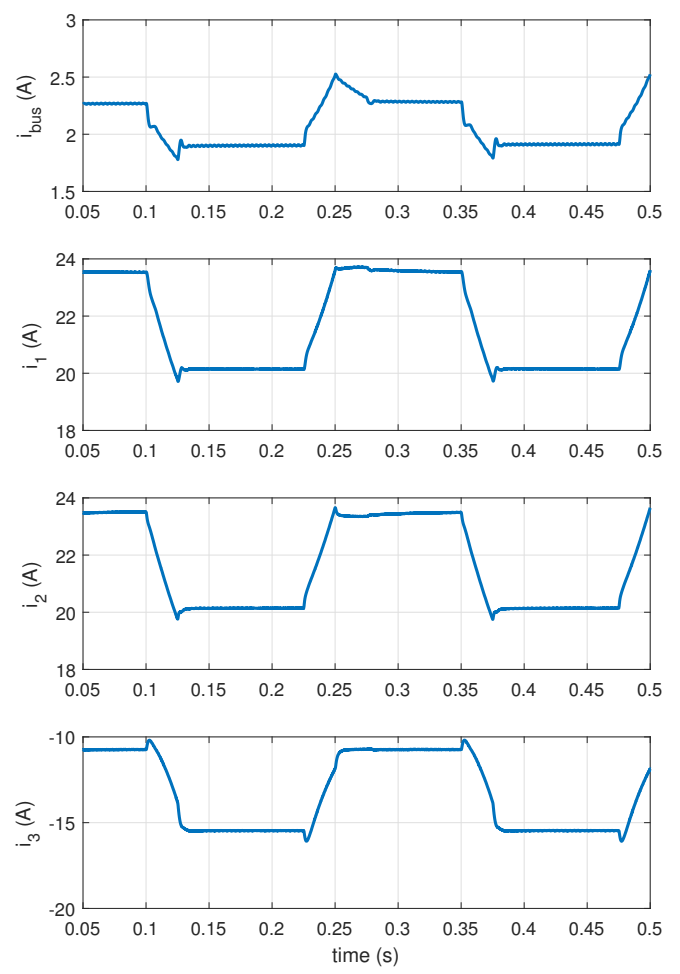

Fig. 7: Simulation results of the system with variable bus voltages.

is the $\mathrm{LV}$ voltage $v_{L V}$. A proportional integral (PI) regulator selects the phase shift $\delta$ of the modulation and regulates the active power. A virtual resistor $R_{v}$ control with time constant $\tau_{R V}$ is used for the power sharing of different converters. The converters are coupled with cables with inductance $L_{1}, L_{2}$, $L_{3}$. In the LV bus a resistive load $R_{\text {load }}$ and a constant power load represented by DAB number 3 .

In order to show that this architecture allows for the seamless control of the bus voltage, a simulation scenario is reported in Fig. 7, where the reference voltages $v_{b u s}^{*}$ and $v_{L V}^{*}$ are varied with a trapezoidal shape from $1 \mathrm{pu}$ to $0.7 \mathrm{pu}$. The voltages and the currents of the converters are shown in the simulation. The constant power load absorbs a power of 280 $\mathrm{W}$ independently of the voltage (the current is negative and it increases in magnitude when the voltage decreases). The resistive load $R_{\text {load }}=0.7 \Omega$ changes its power according to the square of the voltage variation. This simulation explains conceptually how the whole EPDS can be operated with variable DC Link voltage while the power electronics regulates the power.

The effects of this operation mode is that the maximum available power is reduced because of the reduced voltage. However, a reduced DC link voltage reduces the switching losses, so an increase of the current can be realized without impacting on the thermal design.

Although this method can already be adopted in today's aircrafts, in order to minimize the impact there are two main requirements:

- Precise space weather forecast that allows for a good estimate of the secondary dose rate on the aircraft route.

- Precise lifetime models of the devices with a realistic mix of secondary particles.

\section{CONClusion}

This paper reviews the effects of the solar activity on the earth atmosphere and draws a connection to the power electronics of the electrical power distribution system of the more electric aircraft. Generation of secondary particles, especially neutrons, due to solar activity, can cause failure in the power devices, forcing the power system or the converter to be reconfigured after the fault occurrence. A possibility to decrease the chance of a failure is to perform the voltage regulation of the whole distribution system in response of a dangerous space weather forecast. The reduction of the DC Link voltage of the power converters results in an exponential lowering of the failure probability at the expense of a reduced power capability. However, a reduced DC link voltage reduces the switching losses as well and this allows for a higher current capability that partially compensates for the reduction of the maximum power. An example simulation shows the feasibility of the proposed approach, where the power electronics still 
regulates a constant power even in response to a bus voltage variation.

\section{ACKNOWLEDGMENT}

This work was supported by the Ningbo Science \& Technology Beauro under Grant 2013A31012 and Grant 2014A35007.

\section{REFERENCES}

[1] A. A. Pacini, "Cosmic rays: bringing messages from the sky to the Earth's surface," Revista Brasileira de Ensino de FÂsica, vol. 39, 00 2017. [Online]. Available: http://www.scielo.br/scielo.php?script=sci arttext\&pid=S1806-11172017000100406\&nrm=iso

[2] J. A. Miller, P. J. Cargill, A. G. Emslie, G. D. Holman, B. R. Dennis, T. N. LaRosa, R. M. Winglee, S. G. Benka, and S. Tsuneta, "Critical issues for understanding particle acceleration in impulsive solar flares," Journal of Geophysical Research: Space Physics, vol. 102, no. A7, pp. 14631-14659, 1997. [Online]. Available: http://dx.doi.org/10.1029/97JA00976

[3] G. Zank, G. Li, and O. Verkhoglyadova, "Particle acceleration at interplanetary shocks," Space Science Reviews, vol. 130, no. 1-4, pp. 255-272, 2007.

[4] M. Zhang, G. Qin, and H. Rassoul, "Propagation of solar energetic particles in three-dimensional interplanetary magnetic fields," The Astrophysical Journal, vol. 692, no. 1, p. 109, 2009.

[5] L. Desorgher, E. O. Flückiger, and M. Gurtner, "The planetocosmics geant4 application," in 36th COSPAR Scientific Assembly, vol. 36, 2006, p. 2361.

[6] U. Schilling and R. Weiss, "Cosmic ray failures in power electronics," in Semikron AN 17-003, June 2007.

[7] N. Kaminski and A. Kopta, "Failure rates of hipak modules due to cosmic rays," in ABB application note 5SYA 2042-04.

[8] O. C. O. C. Allkofer, P. K. F. Grieder, and M. Fachinformationszentrum Energie, Physik, Cosmic rays on earth. Karlsruhe : Fachinformationszentrum Energie, Physik, Mathematik, 1983, preface also in German.

[9] G. Soelkner, W. Kaindl, H.-J. Schulze, and G. Wachutka, "Reliability of power electronic devices against cosmic radiation-induced failure," Microelectronics Reliability, vol. 44, no. 9, pp. 1399 - 1406, 2004 15th European Symposium on Reliability of Electron Devices, Failure Physics and Analysis. [Online]. Available: http://www.sciencedirect. com/science/article/pii/S0026271404002422

[10] H. R. Zeller, "Cosmic ray failures in power electronics," vol. 38, pp. 2041-2046, 1995.

[11] W. Kaindl, Modellierung hhenstrahlungsinduzierter Ausflle in Halbleiterleistungsbauelementen. University of Munich, PhD Thesis, 2005.

[12] H. Kabza, H. J. Schulze, Y. Gerstenmaier, P. Voss, J. W. W. Schmid, F. Pfirsch, and K. Platzoder, "Cosmic radiation as a cause for power device failure and possible countermeasures," in Proceedings of the 6th International Symposium on Power Semiconductor Devices and Ics, May 1994, pp. 9-12.

[13] C. Felgemacher, S. V. Araujo, C. Noeding, and P. Zacharias, "Benefits of increased cosmic radiation robustness of sic semiconductors in large power-converters," in PCIM Europe 2016; International Exhibition and Conference for Power Electronics, Intelligent Motion, Renewable Energy and Energy Management, May 2016, pp. 1-8.

[14] L. F. Costa, G. Buticchi, and M. Liserre, "A fault-tolerant series-resonant dc-dc converter," IEEE Transactions on Power Electronics, 2016.

[15] G. Buticchi, L. Costa, and M. Liserre, "Improving system efficiency for the more electric aircraft: A look at dcdc converters for the avionic onboard dc microgrid," IEEE Industrial Electronics Magazine, vol. 11, no. 3, pp. 26-36, Sept 2017.

[16] G. Buticchi, L. F. Costa, D. Barater, M. Liserre, and E. Dominguez, "A quadruple active bridge converter for the storage integration on the more electric aircraft," IEEE Transactions on Power Electronics, 2018, in press.

[17] G. Buticchi, L. Costa, and M. Liserre, "Dc/dc conversion solutions to enable smart-grid behavior in the aircraft electrical power distribution system," in IECON 2017 - 43rd Annual Conference of the IEEE Industrial Electronics Society, Oct 2017, pp. 4369-4374.
[18] F. Gao, X. Zheng, and S. Bozhko, "Stability assessment of a droopcontrolled multi-generator electrical power system in the more electric aircraft using parameter space approach," in 2016 IEEE Energy Conversion Congress and Exposition (ECCE), Sept 2016, pp. 1-8.

[19] F. Gao, S. Bozhko, A. Costabeber, G. Asher, and P. Wheeler, "Control design and voltage stability analysis of a droop-controlled electrical power system for more electric aircraft," IEEE Transactions on Industrial Electronics, vol. 64, no. 12, pp. 9271-9281, Dec 2017. 